Article

\title{
Acute Pulmonary Embolism in Patients with and without COVID-19
}

\author{
Antonin Trimaille 1,2 $\mathbb{D}$, Anaïs Curtiaud ${ }^{1}$, Kensuke Matsushita 1,2 $\mathbb{D}$, Benjamin Marchandot ${ }^{1}$ (D), \\ Jean-Jacques Von Hunolstein ${ }^{1} \mathbb{D}$, Chisato Sato ${ }^{1,2}$, Ian Leonard-Lorant ${ }^{3}$, Laurent Sattler $4 \mathbb{D}^{\text {, }}$, Lelia Grunebaum ${ }^{4}$, \\ Mickaël Ohana ${ }^{3}\left(\mathbb{D}\right.$, Patrick Ohlmann ${ }^{1}\left(\mathbb{D}\right.$, Laurence Jesel ${ }^{1,2}$ and Olivier Morel ${ }^{1,2, *}$
}

1 Division of Cardiovascular Medicine, Nouvel Hôpital Civil, Strasbourg University Hospital, 67000 Strasbourg, France; antonin.trimaille@chru-strasbourg.fr (A.T.); anais.curtiaud@chru-strasbourg.fr (A.C.); matsuken_22@yahoo.co.jp (K.M.); benjaminmarchandot@gmail.com (B.M.); jean-jacques.vonhunolstein@chru-strasbourg.fr (J.-J.V.H.); okoge16@gmail.com (C.S.); patrick.ohlmann@chru-strasbourg.fr (P.O.); laurence.jesel@chru-strasbourg.fr (L.J.)

2 INSERM (French National Institute of Health and Medical Research), UMR 1260, Regenerative Nanomedicine, FMTS, 67000 Strasbourg, France

3 Radiology Department, Nouvel Hôpital Civil, Strasbourg University Hospital, 67000 Strasbourg, France; leonard.lorant@gmail.com (I.L.-L.); mickael.ohana@chru-strasbourg.fr (M.O.)

4 Haematology and Haemostasis Laboratory, Centre for Thrombosis and Haemostasis, Nouvel Hôpital Civil, Strasbourg University Hospital, 67000 Strasbourg, France; laurent.sattler@chru-strasbourg.fr (L.S.); lelia.grunebaum@chru-strasbourg.fr (L.G.)

* Correspondence: olivier.morel@chru-strasbourg.fr

Citation: Trimaille, A.; Curtiaud, A.; Matsushita, K.; Marchandot, B.; Von Hunolstein, J.-J.; Sato, C.; Leonard-Lorant, I.; Sattler, L.; Grunebaum, L.; Ohana, M.; et al. Acute Pulmonary Embolism in Patients with and without COVID-19. J. Clin. Med. 2021, 10, 2045. https:// doi.org/10.3390/jcm10102045

Academic Editor: Luca Costanzo

Received: 10 April 2021

Accepted: 7 May 2021

Published: 11 May 2021

Publisher's Note: MDPI stays neutral with regard to jurisdictional claims in published maps and institutional affiliations.

Copyright: (c) 2021 by the authors Licensee MDPI, Basel, Switzerland. This article is an open access article distributed under the terms and conditions of the Creative Commons Attribution (CC BY) license (https:/ / creativecommons.org/licenses/by/ $4.0 /)$.
Abstract: Introduction. Acute pulmonary embolism (APE) is a frequent condition in patients with COVID-19 and is associated with worse outcomes. Previous studies suggested an immunothrombosis instead of a thrombus embolism, but the precise mechanisms remain unknown. Objective. To assess the determinants and prognosis of APE during COVID-19. Methods. We retrospectively included all consecutive patients with APE confirmed by computed tomography pulmonary angiography hospitalized at Strasbourg University Hospital from 1 March to 31 May 2019 and 1 March to 31 May 2020. A comprehensive set of clinical, biological, and imaging data during hospitalization was collected. The primary outcome was transfer to the intensive care unit (ICU). Results. APE was diagnosed in 140 patients: 59 (42.1\%) with COVID-19, and 81 (57.9\%) without COVID-19. A $812 \%$ reduction of non-COVID-19 related APE was registered during the 2020 period. COVID-19 patients showed a higher simplified pulmonary embolism severity index (sPESI) score (1.15 $\pm 0.76 \mathrm{vs}$. $0.83 \pm 0.83, p=0.019)$ and were more frequently transferred to the ICU $(45.8 \%$ vs. $6.2 \%, p<0.001)$. No difference regarding the most proximal thrombus localization, Qanadli score (8.1 \pm 6.9 vs. $9.0 \pm 7.4$, $p=0.45)$, the proportion of subsegmental $(10.2 \%$ vs. $11.1 \%, p=0.86)$, and segmental pulmonary embolism (35.6\% vs. $24.7 \%, p=0.16$ ) was evidenced between COVID-19 and non-COVID-19 APE. In COVID-19 patients with subsegmental or segmental APE, thrombus was, in all cases (27/27 patients), localized in areas with COVID-19-related lung injuries. Marked inflammatory and prothrombotic biological markers were associated with COVID-19 APE. Conclusions. APE patients with COVID-19 have a particular clinico-radiological and biological profile and a dismal prognosis. Our results emphasize the preeminent role of inflammation and a prothrombotic state in these patients.

Keywords: COVID-19; SARS-CoV-2; thrombosis; acute pulmonary embolism; inflammation; computed tomography pulmonary angiography

\section{Introduction}

Since the outbreak of coronavirus disease 2019 (COVID-19), acute pulmonary embolism (APE) has been recognized as a frequent complication that carried a dismal prognosis [1,2]. Evidence from autopsy series reported endotheliitis [3], pulmonary vascular 
microthrombosis [4], and intense inflammation within the pulmonary vasculature. Laboratory features of hypercoagulability in COVID-19 include marked elevation of D-dimer and fibrinogen level, and the presence of lupus anticoagulant (LA) at high frequency [5-7]. The concept of immunothrombosis has emerged to describe radiological findings of subsegmental or segmental thrombi [8]. Moreover, a decline in incidence of APE and imaging procedures has been reported due to the COVID-19 pandemic [9].

In an effort to better characterize the pathological mechanisms underlying COVID-19 associated APE, we sought to compare COVID-19 and non-COVID-19 APE. The objectives of our study were to compare the difference regarding clinical, biological, and radiological characteristics of COVID-19 and non-COVID-19 patients with APE admitted to the general ward at our institution, and to assess the impact of COVID-19 in APE prognosis.

\section{Methods}

\subsection{Setting and Study Population}

All patients with clinical and/or biological suspicion of APE (D-Dimers elevation) and computed tomography (CT) pulmonary angiography (CTPA) performed in the Radiology departments of Strasbourg University Hospital (two centers: Nouvel Hôpital Civil and Hôpital de Hautepierre) were reviewed for imaging techniques and findings. We retrospectively included all patients $\geq 18$ years of age with CTPA confirmed APE admitted to general wards between 1 March and 31 May of the years 2019 and 2020. Exclusion criteria were: an unclear diagnosis of APE or the absence of APE on CTPA. Anonymized data from all patients were collected via our institution's electronic health record. A complete set of clinical, biological, and imaging data were recorded. The present study was approved by the research ethics committee of Strasbourg Hospital (authorization CE-2020-57) who waived the need of informed consent.

Medical management was left to the discretion of the treating physician. All APE patients were treated with therapeutic anticoagulation according to current guidelines [10]. Non-systematic thromboprophylaxis before the index APE event included standard doses (subcutaneous Enoxaparin at $0.4 \mathrm{~mL}$ per day, subcutaneous Fondaparinux at $2.5 \mathrm{mg}$ per day, or intravenous unfractionated heparin at $200 \mathrm{IU}$ per hour) or an intermediate dose (subcutaneous Enoxaparin at $0.4 \mathrm{~mL}$ twice per day).

\subsection{Study Definitions}

A confirmed case of COVID-19 was defined by a positive result of a reverse-transcripta se-polymerase-chain-reaction (RT-PCR) assay of a specimen collected on a nasopharyngeal swab and by typical findings of COVID-19 at chest CT (bilateral and peripheral ground glass opacities and/or alveolar consolidations) [11]. Venous thromboembolism (VTE) risk was assessed on admission to hospital via the Padua Prediction Score and the IMPROVE score [12-15]. The simplified pulmonary embolism severity index (sPESI) score, a risk stratification tool to determine the mortality of patients with newly diagnosed APE, was calculated for every patient at the time of APE diagnosis [16].

\subsection{Imaging}

APE was diagnosed either at the time of admission or during hospital stay by a CTPA acquired on a 64-row or greater scanner, after injection of 50 to $75 \mathrm{~mL}$ of high concentration iodine contrast media. The most proximal localization of thrombus in the pulmonary vasculature was recorded. Total thrombus load was assessed via the Qanadli CT pulmonary obstruction index and calculated on CTPA for all patients [17]. Deep vein thrombosis (DVT), in subjects with signs or symptoms, was diagnosed by a complete duplex ultrasound from thigh to ankle with Doppler waveforms and images. 
Lesions severity of COVID-19 at CT was visually classified following the European Society of Radiology (ESR)/European Society of Thoracic Imaging (ESTI) guidelines [11]. The extent of COVID-19 disease on chest CT scans was assessed as pulmonary injuries extension in percentage of the total pulmonary field and classified as minimal (stage $1<10 \%$ ), moderate (stage $2=10$ to $25 \%$ ), severe (stage $3=25$ to $50 \%$ ), and critical (stage $4>50 \%$ ).

\subsection{Laboratory Tests}

Laboratory values included leukocytes count, platelets count, hemoglobin, and inflammatory markers, such as C-reactive protein (CRP). Hemostasis assays (fibrinogen, D-dimer, lupus anticoagulant (LA) detection) were analyzed on STA- ${ }^{\circledR}$ Evolution (Diagnostica Stago ${ }^{\circledR}$, Asnières-sur-Seine, France) with standard commercial reagents and protocols.

LA detection was based on several tests. First, two screening tests were performed, respectively a Diluted Russel Viper Venom Time (dRVVT screen) made with the STA ${ }^{\circledR}$ Staclot dRVV Screen reagent (Stago), and an activated partial prothromboplastin time (aPTT) performed with the STA ${ }^{\circledR}$-PPT A reagent (Stago). Positivity of one or both screening tests induced a mixing test at 1:1 proportion with a commercial frozen PNP (CRYOcheck ${ }^{\mathrm{TM}}$ Pooled Normal Plasma, CRYOcheck, Montpellier, France). Moreover, a positive dRVVT screen induced a confirmatory test with an increased concentration of phospholipids (dRVVT confirm), performed with the STA ${ }^{\circledR}$-Staclot dRVV Confirm reagent (Stago). dRVVT screen, DRVVT confirm, and aPTT results were expressed as a ratio of patient-to-PNP. Mixing tests results were expressed as an index of circulating anticoagulant (ICA). LA was considered as positive only if the normalized dRVVT ratio (screen ratio/confirm ratio) was $>1.2$ and all causes of false positive were excluded (i.e., anticoagulation conditions).

\subsection{Study Outcomes}

The primary outcome was transfer to the intensive care unit (ICU), which reflected the need for hemodynamic and respiratory support. Secondary outcomes included in-hospital death and the need of mechanical ventilation during hospital stay.

\subsection{Statistical Analysis}

Continuous variables were expressed as mean \pm standard deviation or median and interquartile range as appropriate and categorical variables as counts and percentages. Continuous variables were compared with the use of parametric (Student's $t$ test) or nonparametric Mann-Whitney tests as appropriate. Categorical variables were compared with chi-square test or Fischer's exact test. The time to event was defined as the time from hospital admission to the date of transfer to the ICU, with patients censored at death or end of study. The impact of COVID-19 on prognosis during APE was assessed using both univariate and multivariate Cox hazard model.

A two-tailed $p$ value $<0.05$ was considered significant. Statistical analyses were performed using SPSS 17.0 for Windows (SPSS Inc., Chicago, IL, USA).

\section{Results}

\subsection{Baseline Characteristics}

A total of 8722 chest CT were performed during the 2020 period (from 1-31 March), with a $915 \%$ and $12 \%$ increase, respectively, in chest CT $(n=3573)$ and CTPA $(n=808)$ compared with the equivalent period in 2019 (Figure 1). 


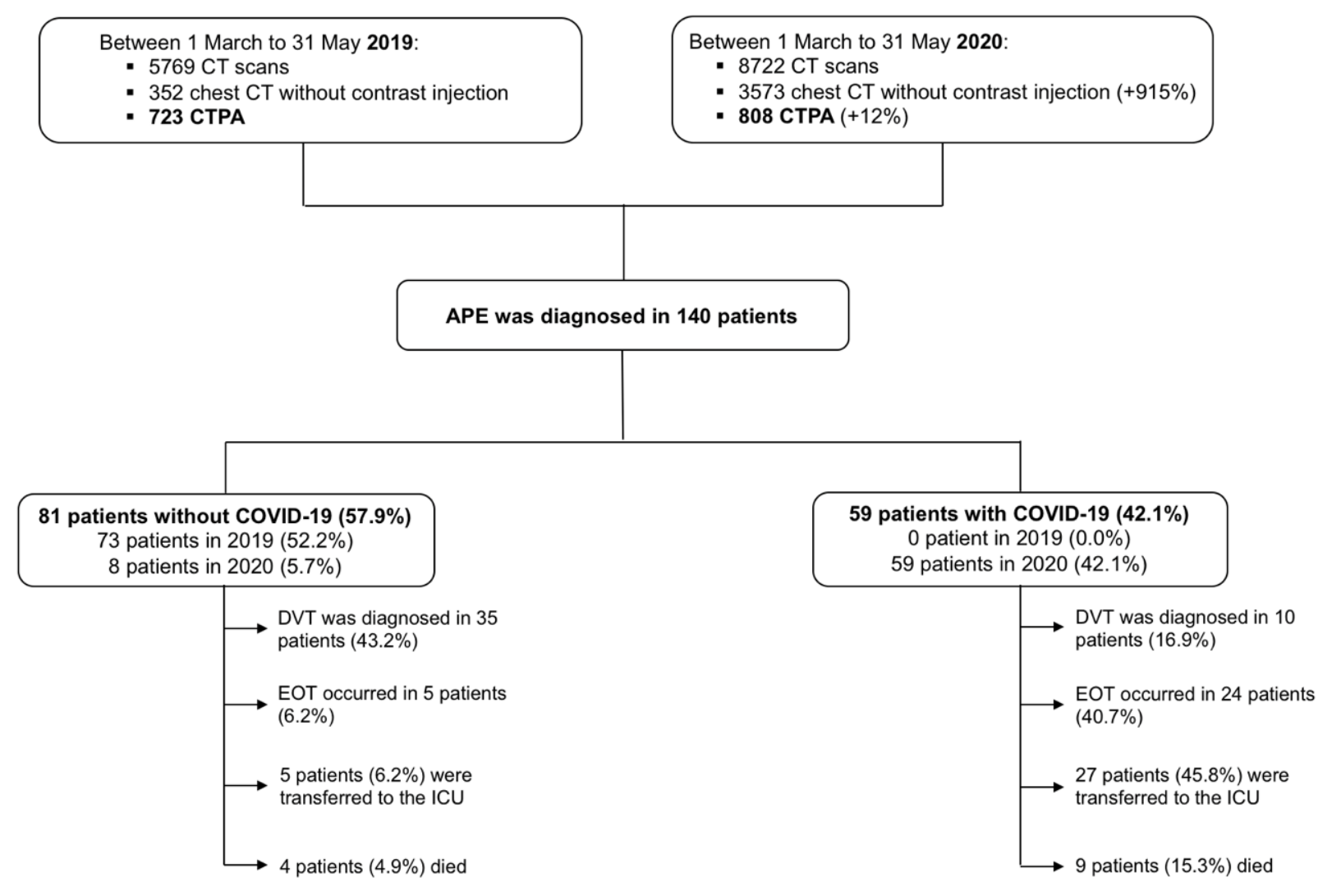

Figure 1. Flow-chart of the study.

APE was diagnosed in 140 patients: 59 patients with COVID-19 and 81 without COVID-19. A total of 8 non COVID-19 related APE was registered during the 2020 period corresponding to an $812 \%$ reduction as compared with 2019. APE was diagnosed in median 3 (interquartile range (IQR) 10) days after admission for COVID-19 patients, and 0 (IQR 1) days for non COVID-19 patients. Imaging test for DVT was performed in 14/59 (23.7\%) patients with COVID-19 and in 63/81 (77.8\%) in patients without COVID-19. COVID19 patients were younger, more frequently male and obese (Table 1). Traditional VTE risk factors such as previous VTE, previous APE and active cancer were less frequently encountered in patients with COVID-19 $(p<0.05)$. Specific VTE risk assessment models via the Padua prediction score and the IMPROVE score, were not significantly different between the two groups. The mean sPESI score was higher in patients with COVID-19 $(1.15 \pm 0.76$ vs. $0.83 \pm 0.83, p=0.019)$ and a lower proportion of these patients had a low sPESI risk $(16.9 \%$ vs. $33.3 \%, p=0.034)$ (Table 2$)$. Significantly more patients with COVID-19 were under standard or intermediate dose of thromboprophylaxis before APE in comparison with patients without COVID-19 (Table 1). 
Table 1. Baseline characteristics.

\begin{tabular}{|c|c|c|c|}
\hline \multicolumn{4}{|c|}{ Patients with Pulmonary Embolism } \\
\hline & $\begin{array}{l}\text { COVID-19 Negative } \\
\quad(n=81)\end{array}$ & $\begin{array}{c}\text { COVID-19 Positive } \\
\quad(n=59)\end{array}$ & $p$ Value \\
\hline \multicolumn{4}{|c|}{ Demographic Characteristics } \\
\hline Age-y & $70.2 \pm 15.1$ & $63.9 \pm 14.4$ & 0.014 \\
\hline Male-n (\%) & $41(50.6)$ & $41(69.5)$ & 0.037 \\
\hline \multicolumn{4}{|c|}{ Cardiovascular risk factors } \\
\hline Obesity-n (\%) & $12(14.8)$ & $21(35.6)$ & 0.005 \\
\hline Hypertension $-n(\%)$ & $51(63.0)$ & $30(50.8)$ & 0.169 \\
\hline Diabetes- $n(\%)$ & $11(13.6)$ & $16(27.1)$ & 0.053 \\
\hline Dyslipidemia-n (\%) & $24(29.6)$ & $19(32.8)$ & 0.713 \\
\hline Smoking- $n(\%)$ & $7(8.6)$ & $3(5.1)$ & 0.519 \\
\hline \multicolumn{4}{|c|}{ Medical history } \\
\hline Previous VTE- $n(\%)$ & $21(25.9)$ & $5(8.5)$ & 0.008 \\
\hline $\mathrm{APE}-n(\%)$ & $6(7.4)$ & $0(0.0)$ & 0.039 \\
\hline DVT $-n(\%)$ & $17(21.0)$ & $5(8.5)$ & 0.059 \\
\hline Heart failure $-n(\%)$ & $1(1.2)$ & $4(6.8)$ & 0.162 \\
\hline $\mathrm{CKD}^{*}-n(\%)$ & $4(4.9)$ & $2(3.4)$ & 1.000 \\
\hline COPD $-n(\%)$ & $5(6.2)$ & $2(3.4)$ & 0.699 \\
\hline Active cancer- $n(\%)$ & $13(16.0)$ & $2(3.4)$ & 0.024 \\
\hline Cancer in remission $-n(\%)$ & $4(4.9)$ & $7(11.9)$ & 0.202 \\
\hline \multicolumn{4}{|c|}{ Medications before hospitalization } \\
\hline OAC-no. $(\%)$ & $9(11.1)$ & $2(3.4)$ & 0.119 \\
\hline SAPT-no. (\%) & $18(22.2)$ & $7(11.9)$ & 0.125 \\
\hline DAPT-no. (\%) & $1(1.2)$ & $1(1.7)$ & 1.000 \\
\hline ACEi-no. $(\%)$ & $7(8.6)$ & $10(16.9)$ & 0.190 \\
\hline ARBs-no. (\%) & $26(32.1)$ & $11(18.6)$ & 0.084 \\
\hline Beta-blocker-no. (\%) & $19(23.5)$ & $14(23.7)$ & 1.000 \\
\hline Statins-no. $(\%)$ & $20(24.7)$ & $11(18.6)$ & 0.419 \\
\hline Oral contraceptives-no. (\%) & $2(2.5)$ & $0(0.0)$ & 0.509 \\
\hline \multicolumn{4}{|c|}{ VTE risk assessment } \\
\hline Padua score $\geq 4-n(\%)^{\dagger}$ & $81(100)$ & $59(100)$ & 1.000 \\
\hline IMPROVE score $-n(\%) \ddagger$ & $1.9 \pm 1.6$ & $1.4 \pm 0.9$ & 0.060 \\
\hline \multicolumn{4}{|c|}{ Thromboprophylaxis before VTE } \\
\hline None- $n(\%)$ & $68(84.0)$ & $28(47.5)$ & $<0.001$ \\
\hline Standard dose $-n(\%)$ & $5(6.2)$ & $21(35.6)$ & $<0.001$ \\
\hline Intermediate dose $-n(\%)$ & $0(0.0)$ & $5(8.5)$ & 0.012 \\
\hline Therapeutic dose $-n(\%)$ & $7(8.6)$ & $5(8.5)$ & 0.611 \\
\hline \multicolumn{4}{|c|}{ Outcomes during hospitalization } \\
\hline Transfer to ICU- $n(\%)$ & $5(6.2)$ & $27(45.8)$ & $<0.001$ \\
\hline $\begin{array}{l}\text { Need for mechanical } \\
\text { ventilation- } n(\%)\end{array}$ & $5(6.2)$ & $24(40.7)$ & $<0.001$ \\
\hline In-hospital death- $n(\%)$ & $4(4.9)$ & $9(15.3)$ & 0.073 \\
\hline DVT $-n(\%) \S$ & $35(43.2)$ & $10(16.9)$ & 0.001 \\
\hline Length of stay-days & $12.1 \pm 13.4$ & $15.5 \pm 7.7$ & 0.142 \\
\hline
\end{tabular}

Data are presented as mean \pm standard deviation in case of any other indication. ${ }^{*}$ Chronic kidney disease is defined by eGFR $\leq 60 \mathrm{~mL} / \mathrm{min} / 1.73 \mathrm{~m}^{2} .{ }^{\dagger}$ Venous thromboembolism risk was evaluated on admission to hospital via the Padua Prediction Score. $¥$ Venous thromboembolism risk was also evaluated on admission to hospital via the Improve VTE risk score. \& Imaging test for DVT was performed in 63 in patients without COVID-19 and in 14 patients with COVID-19. Abbreviations: ACEi, angiotensin converting enzyme inhibitor; ARBs, angiotensin-II receptor blockers; APE, acute pulmonary embolism; BMI, body mass index; CKD, chronic kidney disease; COPD, chronic obstructive pulmonary disease; COVID-19, coronavirus disease 2019; DAPT, dual antiplatelet therapy; DVT, deep vein thrombosis; ICU, intensive care medicine, OAC, oral anticoagulant; SAPT, single antiplatelet therapy; VTE, venous thromboembolism. 
Table 2. Characteristics of acute pulmonary embolism stratified by the presence or absence of COVID-19.

\begin{tabular}{|c|c|c|c|}
\hline \multicolumn{4}{|c|}{ Patients with Pulmonary Embolism } \\
\hline & $\begin{array}{l}\text { COVID-19 Negative } \\
(n=81)\end{array}$ & $\begin{array}{l}\text { COVID-19 Positive } \\
(n=59)\end{array}$ & $p$ Value \\
\hline \multicolumn{4}{|c|}{ APE Severity } \\
\hline sPESI & $0.83 \pm 0.83$ & $1.15 \pm 0.76$ & 0.019 \\
\hline Low risk- $n(\%)$ & $27(33.3)$ & $10(16.9)$ & 0.034 \\
\hline Intermediate low risk- $n(\%)$ & $36(44.4)$ & $27(47.5)$ & 0.735 \\
\hline Intermediate high risk- $n(\%)$ & $17(21.0)$ & $19(32.2)$ & 0.171 \\
\hline High risk $-n(\%)$ & $1(1.2)$ & $2(3.4)$ & 0.573 \\
\hline \multicolumn{4}{|c|}{ APE localization } \\
\hline Sub-segmental- $n(\%)$ & $9(11.1)$ & $6(10.2)$ & 0.859 \\
\hline Segmental- $n(\%)$ & $20(24.7)$ & $21(35.6)$ & 0.162 \\
\hline Lobar- $n(\%)$ & $26(32.1)$ & $15(25.4)$ & 0.391 \\
\hline Troncular- $n(\%)$ & $26(32.1)$ & $17(28.8)$ & 0.677 \\
\hline $\begin{array}{l}\text { Co-localization between } \\
\text { segmental or subsegmental } \\
\text { thrombus and COVID-19 } \\
\text { related lung injuries- } n(\%)\end{array}$ & - & $27(100)$ & - \\
\hline \multicolumn{4}{|c|}{ Thrombus load assessment } \\
\hline Qanadli score-IU & $9.0 \pm 7.4$ & $8.1 \pm 6.9$ & 0.452 \\
\hline
\end{tabular}

Data are presented as mean \pm standard deviation in case of any other indication. Abbreviations: APE, acute pulmonary embolism; COVID-19, coronavirus disease 2019; sPESI, simplified pulmonary embolism severity index; IU, international units.

\subsection{Imaging of Acute Pulmonary Embolism}

No difference regarding the most proximal thrombus localization within the pulmonary vasculature could be evidenced between COVID-19 and non COVID-19 APE (Table 2). The proportion of subsegmental $(10.2 \%$ vs. $11.1 \%, p=0.86)$ and segmental pulmonary embolism $(35.6 \%$ vs. $24.7 \%, p=0.16)$ was not significantly different in patients with and without COVID-19. Among COVID-19 patients with subsegmental or segmental APE, thrombus was in all cases (27/27 patients) localized in areas with COVID-19 related lung injuries (Figure 2). Total thrombus load assessed via the Qanadli score did not significantly differ between the two subsets of patients $(8.1 \pm 6.9$ in COVID-19 patients vs. $9.0 \pm 7.4$ in non COVID-19 patients, $p=0.45$ ).

\subsection{Biological Phenotype of COVID-19 Related Acute Pulmonary Embolism}

COVID-19 patients at admission showed lower leukocytes count (including eosinophils, basophils, and lymphocytes counts: $p<0.005)$ and higher CRP values $(p=0.005)$ (Table 3$)$. While platelets count (PC) and mean platelet volume (MPV) did not significantly differ between the two groups; the MPV /PC ratio was higher in coronavirus patients $(p=0.023)$. Likewise, abnormal coagulation parameters including activated partial thromboplastin time (aPTT) prolongation and higher fibrinogen were more frequently observed in COVID19 patients $(p<0.005)$. D-Dimer level was lower at admission in COVID-19 patients $(p=0.047)$ whereas no gradual increase nor difference during hospital stay course were observed in the two subsets of patients. A dramatic increase in the incidence of lupus anticoagulant could be evidenced in COVID-19 patients in comparison with non-COVID-19 patients $(82.4 \%$ vs. $12.5 \%$, respectively, $p<0.001)$. During hospitalization, leukocytes peak, platelets peak, CRP, and fibrinogen peaks were significantly higher in patient with COVID$19(p<0.05)$. Renal injury was more frequent in COVID-19 patients with a higher creatinine peak. At discharge, no difference was found in the different blood tests performed. 

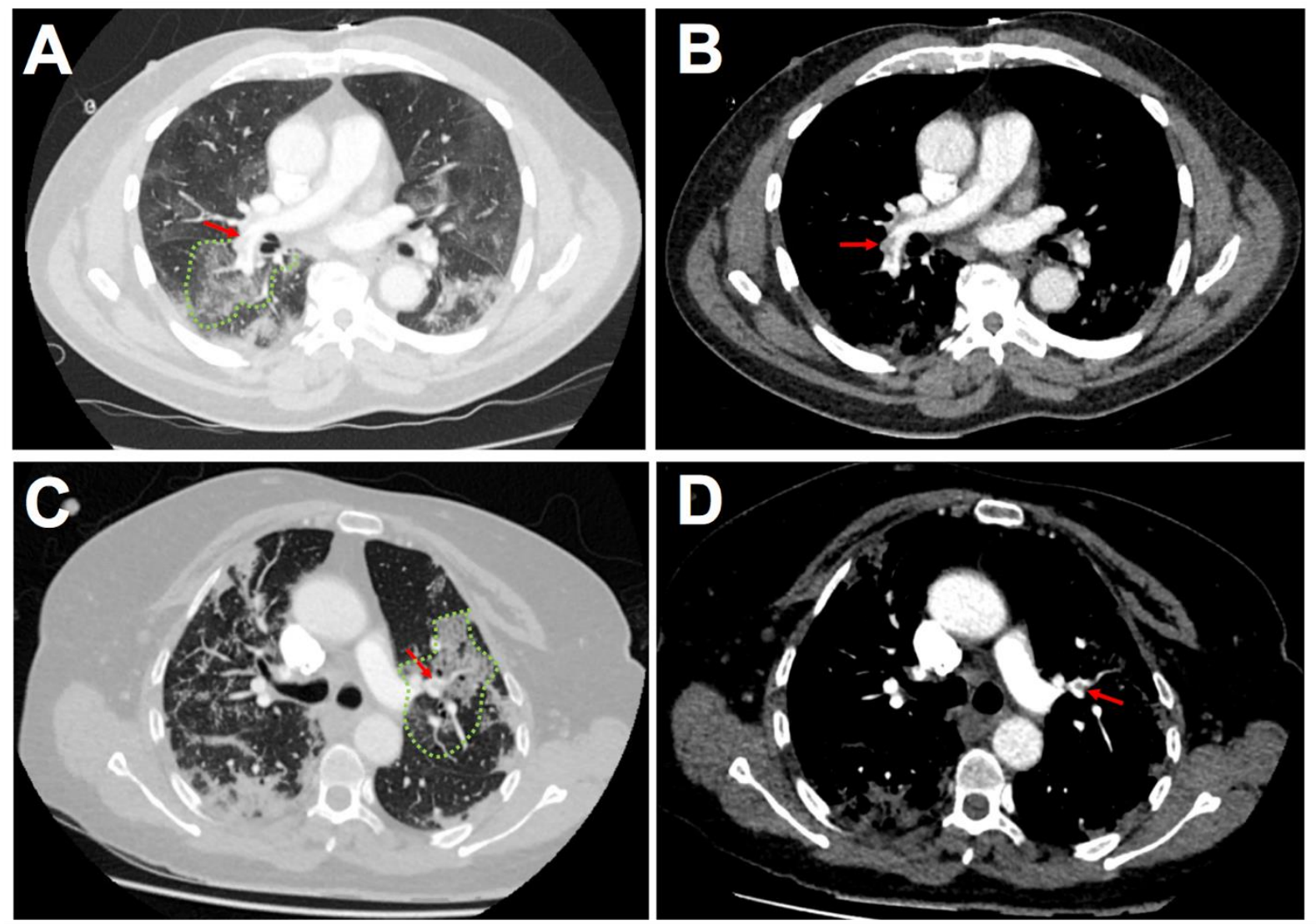

Figure 2. Examples of CTPA of COVID-19 patients with acute pulmonary embolism (lung windows: (A,C); mediastinal windows: (B,D)). Red arrows show lobar and segmental thrombus within the pulmonary vasculature. Green dots: areas with COVID-19 related parenchymal injuries. Abbreviations: COVID-19, coronavirus disease 2019; CTPA, computed tomography pulmonary angiography.

Table 3. Laboratory findings at admission, during hospitalization, and at discharge.

\begin{tabular}{|c|c|c|c|}
\hline \multicolumn{4}{|c|}{ Patients with Pulmonary Embolism } \\
\hline & $\begin{array}{l}\text { COVID-19 Negative } \\
\quad(n=81)\end{array}$ & $\begin{array}{c}\text { COVID-19 Positive } \\
\qquad(n=59)\end{array}$ & $p$ Value \\
\hline \multicolumn{4}{|c|}{ At Admission } \\
\hline Leukocytes $-\times 10^{9}$ per $\mathrm{L}$ & $10.26 \pm 3.48$ & $9.00 \pm 3.95$ & 0.048 \\
\hline Neutrophils $-\times 10^{9}$ per $\mathrm{L}$ & $7.49 \pm 3.26$ & $7.14 \pm 3.59$ & 0.554 \\
\hline Eosinophils $-\times 10^{9}$ per L & $0.14 \pm 0.14$ & $0.03 \pm 0.05$ & $<0.001$ \\
\hline Basophils $-\times 10^{9}$ per $\mathrm{L}$ & $0.05 \pm 0.04$ & $0.02 \pm 0.02$ & $<0.001$ \\
\hline Lymphocytes $-\times 10^{9}$ per $\mathrm{L}$ & $1.59 \pm 1.19$ & $1.09 \pm 0.53$ & 0.003 \\
\hline Monocytes $-\times 10^{9}$ per $\mathrm{L}$ & $0.80 \pm 0.34$ & $0.69 \pm 0.43$ & 0.121 \\
\hline Hemoglobin-g/dL & $12.4 \pm 2.4$ & $13.4 \pm 2.2$ & 0.008 \\
\hline Platelets $-\times 10^{9}$ per $\mathrm{L}$ & $266 \pm 130$ & $231 \pm 99$ & 0.088 \\
\hline MPV-fL & $9.9 \pm 10.5$ & $10.3 \pm 10.9$ & 0.079 \\
\hline $\mathrm{MPV} /$ Platelets ratio-IU & $4.1 \pm 5.3$ & $4.9 \pm 7.4$ & 0.023 \\
\hline Creatinine- $\mu \mathrm{mol} / \mathrm{L}$ & $77.1 \pm 29.3$ & $83.1 \pm 45.0$ & 0.349 \\
\hline $\mathrm{eGFR}-\mathrm{mL} / \mathrm{min} / 1.73 \mathrm{~m}^{2}$ & $79 \pm 23$ & $84 \pm 23$ & 0.162 \\
\hline $\mathrm{CRP}-\mathrm{mg} / \mathrm{L}$ & $63.7 \pm 66.2$ & $99.5 \pm 78.1$ & 0.005 \\
\hline Albumin-g/L & $36.5 \pm 8.2$ & $34.7 \pm 9.0$ & 0.336 \\
\hline Troponin-ng/L & $203.6 \pm 543.9$ & $384.0 \pm 2124.3$ & 0.506 \\
\hline
\end{tabular}


Table 3. Cont.

\begin{tabular}{|c|c|c|c|}
\hline & \multicolumn{2}{|c|}{ Patients with Pulmonary Embolism } & \multirow[b]{2}{*}{$p$ Value } \\
\hline & $\begin{array}{l}\text { COVID-19 Negative } \\
(n=81)\end{array}$ & $\begin{array}{l}\text { COVID-19 Positive } \\
(n=59)\end{array}$ & \\
\hline \multicolumn{4}{|c|}{ At Admission } \\
\hline $\mathrm{BNP}-\mathrm{pg} / \mathrm{mL}$ & $218 \pm 357$ & $280 \pm 616$ & 0.497 \\
\hline PT-\% & $85 \pm 15$ & $85 \pm 19$ & 0.781 \\
\hline INR-IU & $1.2 \pm 0.4$ & $1.3 \pm 1.2$ & 0.291 \\
\hline aPTT-IU & $1.0 \pm 0.2$ & $1.2 \pm 0.3$ & 0.002 \\
\hline Fibrinogen-g/L & $5.0 \pm 1.6$ & $6.2 \pm 2.0$ & 0.002 \\
\hline D-dimer-ng/mL & $7389 \pm 6736$ & $4738 \pm 5628$ & 0.047 \\
\hline \multicolumn{4}{|l|}{ D-Dimer staging ${ }^{*}-n(\%)$} \\
\hline$<3$ ULN & $8(15.7)$ & $13(31.7)$ & \multirow{3}{*}{0.023} \\
\hline 3-6 ULN & $10(19.6)$ & $11(26.8)$ & \\
\hline$>6 \mathrm{ULN}$ & $33(64.7)$ & $17(41.5)$ & \\
\hline $\mathrm{PO}_{2}-\mathrm{mmHg}$ & $85 \pm 34$ & $88 \pm 35$ & 0.684 \\
\hline $\mathrm{PCO}_{2}-\mathrm{mmHg}$ & $36 \pm 7$ & $36 \pm 9$ & 0.912 \\
\hline $\mathrm{PaO}_{2} / \mathrm{FiO}_{2}$ ratio-IU & $328 \pm 122$ & $266 \pm 106$ & 0.015 \\
\hline Lactate-mmol/L & $1.4 \pm 1.1$ & $1.3 \pm 0.5$ & 0.730 \\
\hline \multicolumn{4}{|c|}{ During Hospitalization } \\
\hline Leukocytes peak $-\times 10^{9}$ per $\mathrm{L}$ & $10.61 \pm 4.15$ & $12.77 \pm 7.39$ & 0.030 \\
\hline Hemoglobin nadir-g/dL & $11.5 \pm 2.3$ & $12.4 \pm 2.2$ & 0.015 \\
\hline Platelets peak $-\times 10^{9}$ per $\mathrm{L}$ & $265 \pm 129$ & $343 \pm 176$ & 0.003 \\
\hline Creatinine peak- $\mu \mathrm{mol} / \mathrm{L}$ & $90.0 \pm 39.8$ & $120.7 \pm 103.4$ & 0.018 \\
\hline CRP peak-mg/L & $74.1 \pm 80.1$ & $162.0 \pm 106.5$ & $<0.001$ \\
\hline Fibrinogen peak-g/L & $5.1 \pm 1.9$ & $7.3 \pm 2.1$ & $<0.001$ \\
\hline D-dimer peak-ng/mL & $7570 \pm 6812$ & $7168 \pm 6595$ & 0.771 \\
\hline \multicolumn{4}{|l|}{ D-Dimer staging ${ }^{*}-n(\%)$} \\
\hline$<3 \mathrm{ULN}$ & $9(18.0)$ & $7(15.6)$ & \multirow{3}{*}{0.933} \\
\hline 3-6 ULN & $8(16.0)$ & $10(22.2)$ & \\
\hline$>6 \mathrm{ULN}$ & $33(66.0)$ & $28(62.2)$ & \\
\hline $\begin{array}{l}\text { Lupus anticoagulant } \\
\text { positive-\% * }\end{array}$ & $1(12.5)$ & $28(82.4)$ & $<0.001$ \\
\hline \multicolumn{4}{|c|}{ At Discharge } \\
\hline Leukocytes $-\times 10^{9}$ per $\mathrm{L}$ & $15.62 \pm 59.95$ & $8.89 \pm 5.38$ & 0.450 \\
\hline Hemoglobin-g/dL & $11.8 \pm 2.5$ & $13.4 \pm 14.1$ & 0.377 \\
\hline Platelets $-\times 10^{9}$ per $\mathrm{L}$ & $287 \pm 138$ & $336 \pm 139$ & 0.065 \\
\hline $\mathrm{CRP}-\mathrm{mg} / \mathrm{L}$ & $51.7 \pm 69.7$ & $36.5 \pm 49.7$ & 0.222 \\
\hline Fibrinogen-g/L & $5.3 \pm 1.9$ & $6.2 \pm 2.0$ & 0.218 \\
\hline \multicolumn{4}{|c|}{$\begin{array}{l}\text { Data are presented as mean } \pm \text { standard deviation. }{ }^{*} \text { D-Dimer level at admission was available in } 51 \text { patients } \\
\text { without COVID-19 and } 41 \text { patients with COVID-19. }{ }^{*} \text { Peak D-Dimer level was available in } 50 \text { patients without } \\
\text { COVID-19 and } 45 \text { patients with COVID-19. }{ }^{*} \text { Lupus anticoagulant was tested in } 8 \text { patients without COVID-19 } \\
\text { and in } 34 \text { patients with COVID-19. Abbreviations: aPTT, activated partial thromboplastin time ratio; BNP, B-type } \\
\text { natriuretic peptide; CRP, C-reactive protein; eGFR, estimated glomerular filtration rate; FiO }{ }_{2} \text {, Fraction of inspired } \\
\text { oxygen; INR, international normalized ratio; IU, international units; } \mathrm{PCO}_{2} \text {, partial pressure of carbon dioxide; } \\
\mathrm{PO}_{2} \text {, partial pressure of oxygen; PT, Prothrombin time; VTE, venous thromboembolism. }\end{array}$} \\
\hline
\end{tabular}

\subsection{Outcomes}

Transfer to the ICU occurred more frequently in patients with COVID-19 (45.8\% vs. $6.2 \%, p<0.001)$ as did mechanical ventilation $(40.7 \%$ vs. $6.2 \%, p<0.001)$ (Table 1$)$. There was a trend for a higher in-hospital death rate $(15.3 \%$ vs. $4.9 \%, p=0.07)$ and length of stay $(15.5 \pm 7.7$ vs. $12.1 \pm 13.4, p=0.14)$ in COVID-19 patients in comparison with non-COVID-19 patients.

By univariate analysis, age, previous VTE, sPESI score, creatinine, platelets, leukocytes, CRP, fibrinogen (all values at peak during hospitalization), LA, and COVID-19 were significantly associated with the occurrence of transfer to the ICU in the study population 
(Table 4). Given the collinearity of LA and COVID-19, we built two models for multivariate analysis. In the first model, including all candidates' predictors, except LA, creatinine at peak (hazard ratio (HR) 1.01, confidence interval (CI) 95\% [1.00-1.02], $p=0.011$ ), CRP at peak (HR 1.00, CI95\% [1.00-1.01], $p=0.012$ ) and COVID-19 (HR 4.19, CI95\% [1.27-13.76], $p=0.018)$ were independent predictors of transfer to the ICU in patients with APE. In the second model, including all candidates' predictors, except COVID-19, only CRP at peak (HR 1.01, CI95\% [1.00-1.02], $p=0.029$ ) was independently associated with transfer to the ICU.

Table 4. Univariate and multivariate analyses for occurrence of transfer to the intensive care unit in the study population.

\begin{tabular}{|c|c|c|c|c|c|c|}
\hline \multirow{3}{*}{ Variables } & \multicolumn{2}{|c|}{ Univariate Analysis } & \multicolumn{4}{|c|}{ Multivariate Analysis } \\
\hline & \multirow{2}{*}{$\operatorname{HR}[95 \% \mathrm{CI}]$} & \multirow{2}{*}{$p$} & \multicolumn{2}{|c|}{ First Model * } & \multicolumn{2}{|c|}{ Second Model * } \\
\hline & & & HR $[95 \%$ CI] & $p$ & HR $[95 \%$ CI $]$ & $p$ \\
\hline Age & $0.96[0.93-0.98]$ & 0.006 & $0.96[0.92-1.00]$ & 0.057 & $1.01[0.93-1.10]$ & 0.660 \\
\hline BMI & $1.05[0.98-1.12]$ & 0.130 & & & & \\
\hline Previous VTE & $0.114[0.01-0.86]$ & 0.035 & $0.11[0.00-1.52]$ & 0.101 & $0.10[0.00-35.08]$ & 0.108 \\
\hline Diabetes Mellitus & 1.95 [0.77-0.49] & 0.154 & & & & \\
\hline CAD & $0.91[0.23-3.49]$ & 0.893 & & & & \\
\hline LV dysfunction & 5.48 [0.87-34.37] & 0.069 & & & & \\
\hline Atrial Fibrillation & $0.31[0.03-2.56]$ & 0.281 & & & & \\
\hline COPD & $0.54[0.06-4.73]$ & 0.585 & & & & \\
\hline History of cancer & $0.62[0.19-1.99]$ & 0.431 & & & & \\
\hline Lack of thromboprophylaxis & $0.48[0.13-1.73]$ & 0.265 & & & & \\
\hline PE localization & $0.89[0.60-1.33]$ & 0.599 & & & & \\
\hline sPESI & $1.91[1.18-3.10]$ & 0.008 & $1.73[0.89-3.35]$ & 0.102 & $2.54[0.33-19.15]$ & 0.365 \\
\hline Creatinine peak & $1.01[1.00-1.01]$ & 0.001 & 1.01 [1.00-1.02] & 0.011 & $1.02[0.98-1.06]$ & 0.260 \\
\hline Platelet peak & $1.00[1.00-1.00]$ & $<0.001$ & & & & \\
\hline Leukocytes peak & 1.12 [1.03-1.22] & 0.004 & & & & \\
\hline CRP peak & 1.01 [1.00-1.01] & $<0.001$ & 1.00 [1.00-1.01] & 0.012 & 1.01 [1.00-1.02] & 0.029 \\
\hline Fibrinogen peak & 1.99 [1.44-2.75] & $<0.001$ & & & & \\
\hline D-Dimer peak & $1.00[1.00-1.00]$ & 0.738 & & & & \\
\hline Troponin peak & 0.99 [0.98-1.00] & 0.099 & & & & \\
\hline LA & $8.50[1.60-45.12]$ & 0.012 & & & 9.53 [0.38-238.92] & 0.170 \\
\hline Qanadli score & $0.97[0.92-1.03]$ & 0.395 & & & & \\
\hline COVID-19 & $12.82[4.53-36.27]$ & $<0.001$ & 4.19 [1.27-13.76] & 0.018 & & \\
\hline
\end{tabular}

* First model included all candidates' predictors except LA. Second model included all candidates' predictors except COVID-19. Abbreviations: $\mathrm{BMI}$, body mass index; $\mathrm{CAD}$, coronary artery disease; $\mathrm{CI}$, confidence interval; COPD, chronic obstructive pulmonary disease; COVID-19, coronavirus disease 2019; CRP, C-reactive protein; HR, hazard ratio; LA, lupus anticoagulant; LV, left ventricle; PE Pulmonary embolism; sPESI, simplified pulmonary embolism severity index; VTE, venous thromboembolism.

\section{Discussion}

In this temporal analysis of 140 patients with acute pulmonary embolism, we observed a marked decline in non-COVID-19 APE during the first European wave of the pandemic. While there was similar thrombus load and location of the most proximal arterial branch involved in COVID-19 and non-COVID-19 patients, elevated markers of thrombosis and inflammation, and worse outcomes in patients with COVID-19 could be evidenced.

\subsection{Insights from the Comparison between COVID-19 and Non-COVID-19 Patients}

In line with previous studies reporting a decrease in the number of patients presenting to hospitals, because of emergency conditions, such as acute coronary syndrome [18-20], stroke [21] and APE [9], we observed a marked decline in non-COVID-19 APE between 1 March 2020 and 31 May 2020. Despite a significant increase in the use of CTPA imaging, we identified a $812 \%$ reduction in non-COVID-19 APE as compared with 2019. The reasons for this observed decline in traditional APE are likely multifactorial: stay-at-home message by governmental and healthcare institutions, fear of exposure to COVID-19 affected subjects at hospital admission. 
Main features of COVID-19 patients with APE have been described in several studies [1,2,22]. As previously reported [22], we found a rare prevalence of the traditional risk factors in COVID-19 patients. History of venous thromboembolism and cancer, two strong predisposing factors of APE in hospitalized patients [23], were significantly less frequently encountered in patients with COVID-19 and none of them had a history of APE. Conversely, proportions of obesity and male sex were higher in COVID-19 patients. These factors have been established as predisposing factors of severe forms of COVID-19 [24]. Besides lung alveolar cells and vascular endothelium, ACE2 is also expressed in adipocytes and up-regulation of ACE2 has been described in obesity. Enhanced ACE2-mediated viral access and replication in adipose tissue was suggested as an important determinant of inflammatory burden [25].

Numerous studies have emphasized that COVID-19 is associated with coagulation parameters abnormalities [5,7]. In our experience, with respect to biological values measured in non-COVID-19 APE patients, the prothrombotic state observed in coronavirus patients was characterized by higher levels of fibrinogen and a marked increase of lupus anticoagulant incidence. Previous reports have emphasized a high incidence of positive lupus anticoagulant during COVID-19 [6]. While inflammation and CRP were known to interfere with some lupus anticoagulant tests, this high incidence persisted after adjustment for CRP levels [26]. It may be attributed to the strong inflammation and in severe cases to cytokine storms. The prolonged aPTT that we have observed in COVID-19 patients in comparison with non-COVID-19 patients could be explained in part by the higher incidence of lupus anticoagulant and thus should not be a limitation to the use of anticoagulation in the prevention and treatment of VTE, as previously proposed [6].

Surprisingly, we found lower D-Dimer level at admission among COVID-19 patients but no difference at peak with non-COVID-19 in contrast with the results published earlier by van Dam et al. [27]. Two hypothesizes can be raised to explain this difference. First, we could not exclude that a shift in the time course of the thromboembolism process occurred in patients with and without COVID-19 since the median delay between admission and APE diagnosis was longer in COVID-19 patients. In addition, it is likely that inflammatory cytokines such as tumor necrosis factor (TNF) can exert an inhibitory effect on the fibrinolytic system [28]. In line with this view, a previous study that included COVID-19 and non-COVID-19 patients hospitalized in ICU for acute respiratory distress syndrome (ARDS), found that D-Dimer was significantly lower in patients with COVID-19 [29].

Besides the prothrombotic state highlighted in COVID-19, previous studies have reported that thrombus described by CTPA was in the majority of cases segmental or subsegmental during COVID-19 related APE [27,30]. Some authors have suggested that localized immunothrombosis process could contribute to the development of a thrombus within the lung inflammation area [8]. In our study, the thrombus load was calculated from CTPA data using the Qanadli score. No difference could be evidenced between the two subsets of patients. Moreover, the distribution of thrombus localization was homogeneously distributed. As we extensively collected thrombus localization for all patients irrespective of the coronavirus status, we reported a systematic concordance of thrombus localization in subsegmental or segmental pulmonary artery and lung segments with COVID-19 related parenchymal injuries. Such findings support the importance of local pulmonary injuries and inflammation as key events in the thrombus growth [27].

In the field of immunothrombosis, circulating platelets have been recognized as the primary cells regulating hemostasis and thrombosis [31]. By expression of different receptors of immune response, such as toll-like receptors (TLR) or NOD-like receptors, platelets have the capacity to recognize viral pathogens [32]. The subsequent platelet activation leads to both inflammatory and prothrombotic response. A previous study showed that COVID-19 induced deep functional modifications in platelets [33]. In our study, while PC and MPV were no different, the MPV/PC ratio, a surrogate marker of platelet activation and function [34], was significantly increased in patients with COVID-19. 
Consistent with this analysis, an increased MPV/PC ratio has been reported as a risk factor of arterial and venous thrombosis [35].

\subsection{Clinical Implications}

On top of high reported incidence, APE is associated with higher transfer to the ICU and in-hospital death during COVID-19 [1,2]. In our study, COVID-19 patients showed worse prognosis, with more frequent ICU transfer and mechanical ventilation. APE prevention is of paramount importance leading to a prompt response of international medical societies to prevent thrombotic events in COVID-19 patients [36]. In line with previous reports [37,38], we found that a sizeable proportion of APE occurred in patients under preventive anticoagulation (35.6\%) while it was very rare in patients without COVID$19(6.2 \%)$. Some reports have found that therapeutic anticoagulation during hospital stay was associated with improved survival among COVID-19 patients $[39,40]$. The results from ongoing trials including the CORIMMUNO-COAG (NCT04344756), COVID-HEP (NCT04345848), and REMAP-CAP (NCT2735707) trials should further clarify this issue.

As recently highlighted in the American guidelines on VTE management in hospitalized patients, VTE risk assessment is a crucial issue [15]. Interestingly, while patients with and without COVID-19 did not share the same risk factors, the VTE prediction risk assessment via the Padua prediction score and the IMPROVE score did not differ between both patient types. It must be noted that all patients in our cohort had a Padua prediction score $\geq 4$ translating a high risk of VTE. As no reliable, applicable, and well define scoring system currently exists to predict VTE in COVID-19, both the Padua and the IMPROVE score seem to well capture patients at risk of VTE, and might help to guide clinician decisions pending additional studies.

\subsection{Study Limitations}

We acknowledge several limitations. The retrospective nature of the study limits the generalizability of the findings. We cannot exclude selection bias in the comparison between patients with and without COVID-19. However, we used a control cohort of patients hospitalized for APE during the same period (1 March to 31 May) of the year 2019 to avoid any seasonality in APE causes. Both execution and frequency of DVT imaging tests were left to the discretion of the treating physician and this may have underestimated its prevalence. The low incidence of DVT in COVID-19 patients appears as a supplementary argument for an immunothrombosis process within the pulmonary vasculature in COVID19 related APE rather than thrombus embolism, but his should be interpreted with caution with regard to different proportion of patients with DVT imaging. Finally, we did not use specific outcomes such as need for inotropic support. We used transfer to the ICU as primary outcome because it reflects both the need of hemodynamic support (inotropic support but also cardiocirculatory assistance) and respiratory support.

\section{Conclusions}

In addition to a marked decline of non-COVID-19 APE during the first wave of coronavirus pandemic in the year 2020, our temporal analysis of 140 patients with APE found a prothrombotic state, several markers of immunothrombosis, and worse outcomes in patients with COVID-19. These data reinforce the need to an efficient risk assessment and prevention of VTE during COVID-19.

Author Contributions: Conceptualization, A.T., B.M., M.O., O.M.; Methodology, A.T., K.M., B.M., M.O., L.J., O.M.; Software, O.M.; Validation, L.S., L.G., M.O., P.O., L.J., O.M.; Formal Analysis, M.O., P.O., L.J., O.M.; Investigation, A.T., A.C., K.M., B.M., M.O., L.J., O.M.; Data Curation, A.T., A.C., K.M., B.M., J.-J.V.H., C.S., I.L.-L., L.S., L.G., M.O., P.O., L.J., O.M.; Writing-Original Draft Preparation, A.T., B.M., O.M., Writing—Review \& Editing, A.T., A.C., K.M., B.M., J.-J.V.H., C.S., I.L.-L., L.S., L.G., M.O., P.O., L.J., O.M.; Supervision, M.O., O.M.; Funding Acquisition, O.M. All authors have read and agreed to the published version of the manuscript. 
Funding: This work was supported by GERCA (Groupe pour l'Enseignement, la prévention et la Recherche Cardiologique en Alsace).

Institutional Review Board Statement: The present study was approved by the research ethics committee of Strasbourg Hospital (authorization CE-2020-57).

Informed Consent Statement: The research ethics committee of Strasbourg Hospital waived the need of informed consent.

Data Availability Statement: The data presented in this study are available on request from the corresponding author.

Acknowledgments: The authors would like to formally acknowledge the commitment of all frontline healthcare workers during the COVID-19 crisis in the University Hospital of Strasbourg as they commit themselves to difficult work and also put themselves at risk of infection. Strasbourg's University Hospital is proud of the commitment of all nurses, including newly graduated nurses, medical students, health support staff, and volunteers.

Conflicts of Interest: The authors declare no conflict of interest.

\section{References}

1. Klok, F.A.; Kruip, M.; van der Meer, N.; Arbous, M.S.; Gommers, D.M.; Kant, K.M.; Kaptein, F.H.J.; van Paassen, J.; Syals, M.A.M.; Huisman, M.V.; et al. Incidence of thrombotic complications in critically ill ICU patients with COVID-19. Thromb. Res. 2020, 191, 145-147. [CrossRef]

2. Trimaille, A.; Curtiaud, A.; Marchandot, B.; Matsushita, K.; Sato, C.; Leonard-Lorant, I.; Sattler, L.; Grunebaum, L.; Ohana, M.; Von Hunolstein, J.J.; et al. Venous thromboembolism in non-critically ill patients with COVID-19 infection. Thromb. Res. 2020, 193, 166-169. [CrossRef]

3. Varga, Z.; Flammer, A.J.; Steiger, P.; Haberecker, M.; Andermatt, R.; Zinkernagel, A.S.; Mehra, M.R.; Schuepbach, R.A.; Ruschitzka, F.; Mochet, H. Endothelial cell infection and endotheliitis in COVID-19. Lancet 2020, 395, 1417-1418. [CrossRef]

4. Ciceri, F.; Beretta, L.; Scandroglio, A.M.; Colombo, S.; Landoni, G.; Ruggeri, A.; Peccatori, J.; D’Angelo, A.; De Cobelli, F.; Rovere-Querini, P.; et al. Microvascular COVID-19 lung vessels obstructive thromboinflammatory syndrome (MicroCLOTS): An atypical acute respiratory distress syndrome working hypothesis. Crit. Care Resusc. 2020, 22, 95-97. [PubMed]

5. Marchandot, B.; Sattler, L.; Jesel, L.; Matsushita, K.; Schini-Kerth, V.; Grunebaum, L.; Morel, O. COVID-19 Related Coagulopathy: A Distinct Entity? J. Clin. Med. 2020, 9, 1651. [CrossRef] [PubMed]

6. Bowles, L.; Platton, S.; Yartey, N.; Dave, M.; Lee, K.; Hart, D.P.; MacDonald, V.; Green, L.; Sivapalaratnam, S.; Pasi, K.J.; et al. Lupus Anticoagulant and Abnormal Coagulation Tests in Patients with Covid-19. N. Engl. J. Med. 2020, 383, 288-290. [CrossRef] [PubMed]

7. Moschonas, I.C.; Tselepis, A.D. SARS-CoV-2 infection and thrombotic complications: A narrative review. J. Thromb. Thrombolysis 2021, 1-13. [CrossRef]

8. Cattaneo, M.; Bertinato, E.M.; Birocchi, S.; Brizio, C.; Malavolta, D.; Manzoni, M.; Muscarella, G.; Orlandi, M. Pulmonary Embolism or Pulmonary Thrombosis in COVID-19? Is the Recommendation to Use High-Dose Heparin for Thromboprophylaxis Justified? Thromb. Haemost. 2020, 120, 1230-1232. [CrossRef]

9. Nopp, S.; Janata-Schwatczek, K.; Prosch, H.; Shulym, I.; Königsbrügge, O.; Pabinger, I.; Ay, C. Pulmonary embolism during the COVID-19 pandemic: Decline in diagnostic procedures and incidence at a university hospital. Res. Pract. Thromb. Haemost. 2020, 4, 835-841. [CrossRef]

10. Konstantinides, S.V.; Meyer, G.; Becattini, C.; Bueno, H.; Geersing, G.J.; Harjola, V.P.; Huisman, M.V.; Humbert, M.; Jennings, C.S.; Jiménez, D.; et al. 2019 ESC Guidelines for the diagnosis and management of acute pulmonary embolism developed in collaboration with the European Respiratory Society (ERS). Eur. Heart J. 2020, 41, 543-603. [CrossRef]

11. Revel, M.P.; Parkar, A.P.; Prosch, H.; Silva, M.; Sverzellati, N.; Gleeson, F.; Brady, A. COVID-19 patients and the radiology department-Advice from the European Society of Radiology (ESR) and the European Society of Thoracic Imaging (ESTI). Eur. Radiol. 2020, 30, 4903-4909. [CrossRef]

12. Barbar, S.; Noventa, F.; Rossetto, V.; Ferrari, A.; Brandolin, B.; Perlati, M.; De Bon, E.; Tormene, D.; Pagnan, A.; Prandoni, P. A risk assessment model for the identification of hospitalized medical patients at risk for venous thromboembolism: The Padua Prediction Score. J. Thromb. Haemost. 2010, 8, 2450-2457. [CrossRef] [PubMed]

13. Arcelus, J.I.; Candocia, S.; Traverso, C.I.; Fabrega, F.; Caprini, J.A.; Hasty, J.H. Venous thromboembolism prophylaxis and risk assessment in medical patients. Semin. Thromb. Hemost. 1991, 17 (Suppl. S3), 313-318. [PubMed]

14. Spyropoulos, A.C.; Anderson, F.A.; FitzGerald, G.; Decousus, H.; Pini, M.; Chong, B.H.; Zotz, R.B.; Bergmann, J.F.; Tapson, V.; Froehlich, J.B.; et al. Predictive and associative models to identify hospitalized medical patients at risk for VTE. Chest 2011, 140, 706-714. [CrossRef] 
15. Henke, P.K.; Kahn, S.R.; Pannucci, C.J.; Secemksy, E.A.; Evans, N.S.; Khorana, A.A.; Creager, M.A.; Pradhan, A.D.; American Heart Association Advocacy Coordinating Committee. Call to Action to Prevent Venous Thromboembolism in Hospitalized Patients: A Policy Statement From the American Heart Association. Circulation 2020, 16, e914-e931. [CrossRef]

16. Jiménez, D.; Aujesky, D.; Moores, L.; Gómez, V.; Lobo, J.L.; Uresandi, F.; Otero, R.; Monreal, M.; Muriel, A.; Yusen, R.D.; et al. Simplification of the pulmonary embolism severity index for prognostication in patients with acute symptomatic pulmonary embolism. Arch. Intern. Med. 2010, 170, 1383-1389. [CrossRef] [PubMed]

17. Qanadli, S.D.; El Hajjam, M.; Vieillard-Baron, A.; Joseph, T.; Mesurolle, B.; Oliva, V.L.; Barré, O.; Bruckert, F.; Dubourg, O.; Lacombe, P. New CT index to quantify arterial obstruction in pulmonary embolism: Comparison with angiographic index and echocardiography. Am. J. Roentgenol. 2001, 176, 1415-1420. [CrossRef]

18. Bhatt, A.S.; Moscone, A.; McElrath, E.E.; Varshney, A.S.; Claggett, B.L.; Bhatt, D.L.; Januzzi, J.L.; Butler, J.; Adler, D.S.; Solomon, S.D.; et al. Fewer Hospitalizations for Acute Cardiovascular Conditions During the COVID-19 Pandemic. J. Am. Coll. Cardiol. 2020, 76, 280-288. [CrossRef]

19. De Rosa, S.; Spaccarotella, C.; Basso, C.; Calabrò, M.P.; Curcio, A.; Filardi, P.P.; Mancone, M.; Mercuro, G.; Muscoli, S.; Nodari, S.; et al. Reduction of hospitalizations for myocardial infarction in Italy in the COVID-19 era. Eur. Heart J. 2020, 41, 2083-2088. [CrossRef]

20. Matsushita, K.; Hess, S.; Marchandot, B.; Sato, C.; Truong, D.P.; Kim, N.T.; Weiss, A.; Jesel, L.; Ohlmann, P.; Morel, O. Clinical features of patients with acute coronary syndrome during the COVID-19 pandemic. J. Thromb. Thrombolysis 2020, 1-10. [CrossRef]

21. Kansagra, A.P.; Goyal, M.S.; Hamilton, S.; Albers, G.W. Collateral Effect of COVID-19 on Stroke Evaluation in the United States. N. Engl. J. Med. 2020, 383, 400-401. [CrossRef]

22. Fauvel, C.; Weizman, O.; Trimaille, A.; Mika, D.; Pommier, T.; Pace, N.; Douair, A.; Barbin, E.; Fraix, A.; Bouchot, O.; et al. Pulmonary embolism in COVID-19 patients: A French multicentre cohort study. Eur. Heart J. 2020, 41, 3058-3068. [CrossRef] [PubMed]

23. Crous-Bou, M.; Harrington, L.B.; Kabrhel, C. Environmental and Genetic Risk Factors Associated with Venous Thromboembolism. Semin. Thromb. Hemost. 2016, 42, 808-820. [CrossRef]

24. Guzik, T.J.; Mohiddin, S.A.; Dimarco, A.; Patel, V.; Savvatis, K.; Marelli-Berg, F.M.; Madhur, M.S.; Tomaszewski, M.; Maffia, P.; D'Acquisto, F.; et al. COVID-19 and the cardiovascular system: Implications for risk assessment, diagnosis, and treatment options. Cardiovasc. Res. 2020, 116, 1666-1687. [CrossRef] [PubMed]

25. Vaughan, C.J.; Cronin, H.; Ryan, P.M.; Caplice, N.M. Obesity and COVID-19: A Virchow's Triad for the 21st Century. Thromb. Haemost. 2020, 120, 1590-1593. [CrossRef]

26. Reyes Gil, M.; Barouqa, M.; Szymanski, J.; Gonzalez-Lugo, J.D.; Rahman, S.; Billett, H.H. Assessment of Lupus Anticoagulant Positivity in Patients with Coronavirus Disease 2019 (COVID-19). JAMA Netw. Open 2020, 3, e2017539. [CrossRef]

27. Van Dam, L.F.; Kroft, L.J.M.; van der Wal, L.I.; Cannegieter, S.C.; Eikenboom, J.; de Jonge, E.; Huisman, M.V.; Klok, F.A. Clinical and computed tomography characteristics of COVID-19 associated acute pulmonary embolism: A different phenotype of thrombotic disease? Thromb. Res. 2020, 193, 86-89. [CrossRef] [PubMed]

28. Medcalf, R.L. Fibrinolysis, inflammation, and regulation of the plasminogen activating system. J. Thromb. Haemost. 2007, 5, 132-142. [CrossRef]

29. Helms, J.; Tacquard, C.; Severac, F.; Leonard-Lorant, I.; Ohana, M.; Delabranche, X.; Merdji, H.; Clere-Jehl, R.; Schenck, M.; Fagot Gandet, F.; et al. High risk of thrombosis in patients with severe SARS-CoV-2 infection: A multicenter prospective cohort study. Intensive Care Med. 2021, 46, 1089-1098. [CrossRef]

30. Middeldorp, S.; Coppens, M.; van Haaps, T.F.; Foppen, M.; Vlaar, A.P.; Müller, M.C.A.; Bouman, C.C.S.; Beenen, L.F.M.; Kootte, R.S.; Heijmans, J.; et al. Incidence of venous thromboembolism in hospitalized patients with COVID-19. J. Thromb. Haemost. 2020, 18, 1995-2002. [CrossRef] [PubMed]

31. Koupenova, M.; Clancy, L.; Corkrey, H.A.; Freedman, J.E. Circulating Platelets as Mediators of Immunity, Inflammation, and Thrombosis. Circ. Res. 2018, 122, 337-351. [CrossRef]

32. Semple, J.W.; Italiano, J.E.; Freedman, J. Platelets and the immune continuum. Nat. Rev. Immunol. 2011, 11, 264-274. [CrossRef]

33. Manne, B.K.; Denorme, F.; Middleton, E.A.; Portier, I.; Rowley, J.W.; Stubben, C.; Petrey, A.C.; Tolley, N.D.; Guo, L.; Cody, M.; et al. Platelet Gene Expression and Function in COVID-19 Patients. Blood 2020, 136, 1317-1329. [CrossRef] [PubMed]

34. Tavil, Y.; Sen, N.; Yazıcı, H.U.; Hızal, F.; Abac1, A.; Cengel, A. Mean platelet volume in patients with metabolic syndrome and its relationship with coronary artery disease. Thromb. Res. 2007, 120, 245-250. [CrossRef] [PubMed]

35. Guenancia, C.; Hachet, O.; Stamboul, K.; Béjot, Y.; Leclercq, T.; Garnier, F.; Yameogo, N.V.; de Maistre, E.; Cottin, Y.; Lorgis, L. Incremental predictive value of mean platelet volume/platelet count ratio in in-hospital stroke after acute myocardial infarction. Platelets 2017, 28, 54-59. [CrossRef] [PubMed]

36. Marchandot, B.; Trimaille, A.; Curtiaud, A.; Matsushita, K.; Jesel, L.; Morel, O. Thromboprophylaxis: Balancing Evidence and Experience During the COVID-19 Pandemic. J. Thromb. Thrombolysis 2020, 50, 799-808. [CrossRef] [PubMed]

37. Poissy, J.; Goutay, J.; Caplan, M.; Parmentier, A.; Duburcq, T.; Lassalle, F.; Jeanpierre, E.; Rauch, A.; Labreuche, J.; Susen, S. Pulmonary Embolism in COVID-19 Patients: Awareness of an Increased Prevalence. Circulation 2020, 142, 184-186. [CrossRef]

38. Llitjos, J.F.; Leclerc, M.; Chochois, C.; Monsallier, J.M.; Ramakers, M.; Auvray, M.; Merouani, K. High incidence of venous thromboembolic events in anticoagulated severe COVID-19 patients. J. Thromb. Haemost. 2020, 18, 1743-1746. [CrossRef] 
39. Tang, N.; Li, D.; Wang, X.; Sun, Z. Abnormal coagulation parameters are associated with poor prognosis in patients with novel coronavirus pneumonia. J. Thromb. Haemost. 2020, 18, 844-847. [CrossRef] [PubMed]

40. Paranjpe, I.; Fuster, V.; Lala, A.; Russak, A.J.; Glicksberg, B.S.; Levin, M.A.; Charney, A.W.; Narula, J.; Fayad, Z.A.; Bagiella, E.; et al. Association of Treatment Dose Anticoagulation with In-Hospital Survival Among Hospitalized Patients with COVID-19. J. Am. Coll. Cardiol. 2020, 76, 122-124. [CrossRef] 